\title{
Relationship Between Cytokines and The Remodeling of the Structural and Functional State of the Left Ventricle in Patients with Coronary Artery Disease
}

\section{AL Alyavi, ZT Sabirjanova, DK Tulyaganova, DI Radjabova*, SK Nuritdinova and T Khan}

Republican Specialized Scientific-Practical Medical Center for Therapy and Medical Rehabilitation, Tashkent, Uzbekistan

*Corresponding Author: DI Radjabova, Republican Specialized Scientific-Practical Medical Center for Therapy and Medical Rehabilitation, Tashkent, Uzbekistan.

Received: July 02, 2019; Published: July 24, 2019

DOI: $10.31080 /$ ASMS.2019.03.0362

\begin{abstract}
The study was carried out to establish the prognostic value of post-infarction (PI) cardiac remodeling and cytokine imbalance for the development of chronic heart failure (CHF) in patients with coronary artery disease (CAD) with the enrolling 55 patients with the history of Q wave myocardial infarction (MI). For of development of CHF in patients with PI myocardial remodeling the clinical picture, cardio hemodynamic indicators and laboratory markers will allow to identify the maladjusted LV model in the early stages of the disease and to develop new pathogenically based treatment methods for this pathology.

Keywords: Left Ventricular Remodeling; Post Myocardial Infarction; Cytokine; Coronary Artery Disease
\end{abstract}

\section{Introduction}

Coronary heart disease (CHD) is one of the main causes of heart failure, which is accompanied by remodeling of the left ventricular (LV) myocardium [1]. Myocardial remodeling with the loss of cardiomyocytes, the development of interstitial fibrosis, accompanying myocardial hypertrophy and ischemic heart disease, ultimately leads to systolic and diastolic dysfunction of the left ventricular myocardium [21]. However, studies have shown that, despite the improvement in the clinical status of patients and the decrease in cardiovascular risk with the use of blockers of these systems, ischemic heart disease continues to progress $[1,4,6,16]$. It is not clear that there is a possibility of a cardiovascular risk of coronary artery disease [18]. According to the progresses and progresses of the synthesis of pro-inflammatory cytokines, such as tumor necrosis factor- $\alpha$ (TNF- $\alpha$ ), interleukin-1 $\beta$ (IL-1 $\beta$ ), interleukin-6 (IL$6)$ and others, which, in turn, determine myocardium remodeling $[10,11,12,15,19]$.

Pro-inflammatory cytokines play an important role in the progression of the disease [9], mainly influencing LV dysfunction, the intensity of myocardial and vascular remodeling processes by controlling the level of cardiomyocyte apoptosis, which in turn is considered as a fundamental mechanism determining the development of contractile and possibly diastolic myocardial dysfunction $[1,2,5,7,8]$.
The modern concept of the pathogenesis of coronary artery disease accumulates in itself all the above theories based on their complementarity, since one cannot rely on the fact that with one seemingly quite logical concept of pathogenesis one can explain all disorders in a patient with heart failure and moreover solve all problems of therapy, affecting the only mechanism for the development and progression of circulatory failure $[3,4,13,14,16,20]$.

In connection with the above, it seems relevant to study the relationship of cytokine imbalance with the pathogenetic mechanisms of post infarction myocardial remodeling and the determination of their prognostic value during post infarction heart failure in patients with IHD.

\section{Aim}

To establish the prognostic value of post-infarction cardiac remodeling and cytokine imbalance in the clinical course of chronic heart failure in patients with coronary artery disease.

\footnotetext{
Materials and Methods

55 patients were examined, the average age was $54.1 \pm 9.8$ years. Among them, 37 men and 18 women. All patients in the history suffered myocardial infarction with $Q$ of the left ventricle of various localization, from two to seven years. All patients had signs of circulatory insufficiency II-III FC by NYHA. II FC by NYHA - 35 patients, III FC - 20 patients. The control group was included 25 IHD and CHF.
} 
All patients, taking into account the functional class, comorbidity, age, received standard therapy for CHF: ACE inhibitors, $\beta$-blockers, angiotensin receptor antagonists, diuretics, antiplatelet agents. All patients underwent general clinical and biochemical blood tests, standard ECG and special studies.

The processes of cardiac remodeling, ischemic dysfunction of the LV myocardium with the identification of local abnormalities of the contractile function were determined using the EchoCG method on a Samsung medison ultrasound device Accuvix.V20 (Korea) using a sector sensor with a color mode and a pulse-wave, continuous-wave mode with a frequency of $2-4 \mathrm{MHz}$ in standard echocardiographic positions. A set of echocardiographic parameters was determined for each patient: calculation of LV remodeling indices, indicators characterizing its systolic function, and values estimating LV preload.

The study of the content of cytokines, endothelin- 1 and vascular endothelial growth factor was carried out by the enzyme immunoassay using the apparatus Humareader Single (Germany).

The quality of life of all patients was assessed using the University of Minnesota questionnaire, designed specifically for patients with CHF T. Rector, J. Cohn (MLHFQ). The result was evaluated by the sum of points: the best quality of life corresponds to 0 , and the worst - 105 points.

Statistical processing. The results were processed using the statistical data analysis package of the STATISTICA 6.0 program and the Biostat software package.

\section{Results and Discussion}

Features of cardiohemodynamics and clinical course of chronic heart failure II and III FC by NYHA in various options for post-infarction remodeling.

In the study were included 55 patients who had Q myocardial infarction with CHF II and III FC by NYHA. Men prevailed in this group of patients ( $67.2 \%$ of men and $32.7 \%$ of women).

Using the criteria Gottdiener JS., et al. (1994), the following types of LV remodeling were identified: normal geometry, eccentric hypertrophy with LV dilatation, eccentric hypertrophy without LV dilatation, concentric LV hypertrophy (Table 1).

\begin{tabular}{|l|c|c|c|c|}
\hline \multicolumn{1}{|c|}{ Indicators } & $\begin{array}{c}\text { Normal geometry } \\
\text { (n-11) }\end{array}$ & $\begin{array}{c}\text { Eccentric hypertrophy with } \\
\text { dilation (n-14) }\end{array}$ & $\begin{array}{c}\text { Eccentric hypertrophy } \\
\text { without dilation (n-13) }\end{array}$ & $\begin{array}{c}\text { LV concentric } \\
\text { hypertrophy (n-12) }\end{array}$ \\
\hline LVMM & $\begin{array}{c}>200 \text { gr. }-\mathrm{m} \\
<150 \text { gr. }-\mathrm{f}\end{array}$ & $\begin{array}{c}>200 \text { gr. }-\mathrm{m} \\
>150 \text { gr. }-\mathrm{f}\end{array}$ & $\begin{array}{c}>200 \text { gr. }-\mathrm{m} \\
>150 \text { gr. }-\mathrm{f}\end{array}$ & $>200$ gr. $-\mathrm{m}$ \\
$>150$ gr. $-\mathrm{f}$
\end{tabular}

Table 1:Types of left ventricular remodeling in the examined patients.

When analyzing morphometric parameters (LV myocardium mass (LV MM), LV myocardium mass index (LVMMI), sphericity index in systole (ISs), sphericity index in diastole (ISd), relative wall thickness index LV (RVTI), end - diastolic volume (EDVI), end-systolic volume index (ESVI), end-diastolic size index (EDSI)) noted that among patients of FC III, individuals with LV remodeling of eccentric hypertrophy with and without dilatation of its cavity predominate. In this group of patients, the end - diastolic volume is $105 \pm 17 \mathrm{ml}$. In all groups of patients with CHF III FC in comparison with similar indicators of patients with CHF II FC there was a decrease in RVTI $(\mathrm{p}<0.01)$.
Thus, it was established that the result of this structural adjustment is the formation of special LV models - like eccentric hypertrophy with dilatation and without LV dilatation, which is accompanied by worsening clinical features of CHF.

Analysis of central hemodynamic parameters in patients with various types of myocardial remodeling showed that in all studied groups there was a decrease in the ejection fraction (EF), systolic shortening (SS) of LV, end-diastolic LV pressure (EDLVP) and high systolic and diastolic myocardial stress. In concentric remodeling 
and normal LV geometry impairing of the LV diastolic function by relaxation type is combined with a less pronounced increase in pre- and afterloading. While in eccentric hypertrophy with and without dilatation of the $\mathrm{LV}$, the pseudonormal type of diastolic dysfunction correlates with high pre- and afterloads.

These changes are combined with a decrease in the quality of life (MLHFQ scale). The maximum decrease in the quality of life (75.82 points on the MLHFQ scale) was found in eccentric hypertrophy with dilation.

Thus, in patients undergoing Q myocardial infarction, cardiac remodeling is characterized by the formation of eccentric hypertrophy with dilatation of $27.5 \%$ and without $\mathrm{LV}$ dilatation of $23.5 \%$; normal heart geometry in $25 \%$ and concentric hypertrophy in $24 \%$ of cases.

In patients undergoing $Q$ myocardial infarction, a symptom of maladaptive remodeling of the left ventricle, along with characteristic clinical symptoms, are systolic dysfunction, a decrease in the index of the relative thickness of the walls of the left ventricle, and restrictive type of its diastolic dysfunction. Disadaptive model of the left ventricle should be considered as remodeling by type of eccentric hypertrophy with dilation $[1,8,17]$.

When studying the level of cytokines in patients undergoing $Q$ myocardial infarction, an increase in TNF- $\alpha$, IL- 6 levels was noted in comparison with the control (Figure. 1). Intergroup studies re- vealed an increase in TNF- $\alpha$ and IL- 6 in the group of eccentric hypertrophy and $\mathrm{LV}$ dilation.

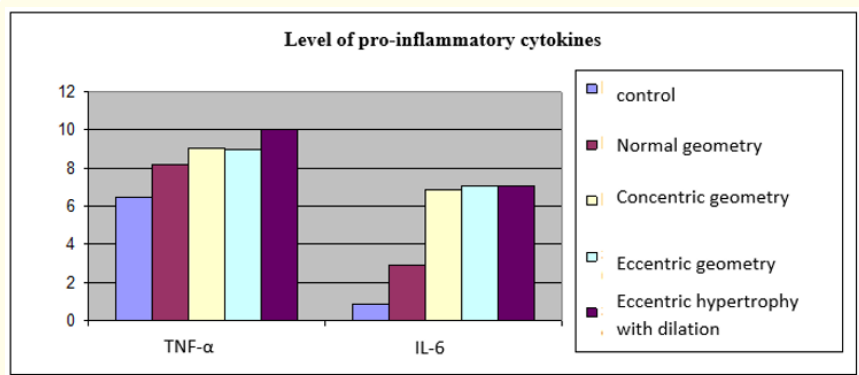

Note: * differences with the normal geometry of the LV p $<0.05$; \# - differences with control $\mathrm{p}<0.05$.

Figure 1: The level of proinflammatory cytokines in the serum.

Thus, a combination of dilatation, myocardial hypertrophy, systolic, diastolic LV dysfunction and negative effects on the myocardium of pro-inflammatory cytokines contributes to the progression of chronic heart failure.

To assess the degree of influence (determination of independent prognostic factors) of the parameters studied on the progression of chronic heart failure and cardio hemodynamic disorders in post infarction myocardial remodeling in patients with FC III by NYHA, multivariate regression analysis was performed (Table 2).

\begin{tabular}{|l|c|c|c|c|}
\hline \multicolumn{1}{|c|}{ Indicators } & $\begin{array}{c}\text { Regression } \\
\text { coefficient - beta }\end{array}$ & Standard error $\boldsymbol{\beta}$ & $\begin{array}{c}\text { Relative risk (95\% of the } \\
\text { confidence interval) }\end{array}$ & P \\
\hline$\Phi B<40, \%$ & 0,52 & 0,1 & $4,3(2,66 ; 8,23)$ & 0,000011 \\
\hline Eccentric hypertrophy with dilation & 0,25 & 0,03 & $2,78(1,2 ; 4,57)$ & 0,0031 \\
\hline TNF - $\boldsymbol{\alpha}$ & 0,08 & 0,04 & $2,3(1,1 ; 3,9)$ & 0,04578 \\
\hline IL-6 & 0,09 & 0,03 & $1,55(0,63 ; 2,61)$ & 0,01093 \\
\hline MLHFQ & 0,2 & 0,05 & $1,75(0,84 ; 2,96)$ & 0,0001 \\
\hline
\end{tabular}

Table 2: Regression coefficients in the multivariate model for predicting chronic heart failure.

The results of the regression analysis showed (Table 2) that the most closely associated with the prognosis of chronic heart failure in patients with FC III in the NYHA was a decrease in $\mathrm{EF}<40 \%$.

The accuracy of prediction of chronic heart failure in postinfarction myocardial remodeling increased with the addition of data on eccentric hypertrophy with dilation, TNF- $\alpha$, data on the decline in the quality of life (MLHFQ scale), and IL-6.
Thus, step-by-step regression multivariate analysis allowed to establish that among the studied parameters of the prognosis of chronic heart failure in patients of FC III by NYHA, the most significant factors were: decrease in $\mathrm{EF}<40 \%$, eccentric hypertrophy with dilation, systolic and diastolic dysfunction of the left ventricle, increase in TNF $\alpha$ and IL-6 $(\mathrm{P}>0.05)$. These changes are combined with a decrease in the quality of life (MLHFQ scale). The maladaptive model, an eccentric hypertrophy with dilation, is also indepen- 
dently associated with the prognosis of the clinical course of postinfarction heart failure.

\section{Conclusion}

Understanding the complex mechanisms of development of chronic heart failure in patients with postinfarction myocardial remodeling in combination with the clinical picture, cardiohemodynamic indicators and laboratory markers will allow not only to identify the maladjusted LV model in the early stages of the disease, but also to develop new pathogenically based treatment methods for this pathology.

\section{Bibliography}

1. Ahmedov I. et al. "Features of hyperreninemic aldosteronism in hypertension patients with metabolic syndrome: 87". European Journal of Preventive Cardiology 23.1 (2016): S16.

2. Alyavi AL. et al. "Efficacy of combined antihypertensive therapy in patients with metabolic syndrome". clinical cardiology 111 river st, hoboken 07030-5774, nj usa: wiley-blackwell, 39 (2016): 12-12.

3. Alyavi A.L. et al. "Combined Therapy with Telmisartan And Amlodipine on Blood Pressure, Echocardiographic Parameters and Microalbuminuria In Patients With Metabolic Syndrome". Journal of Hypertension 36 (2018): e85.

4. Alyavi B., et al. "Influence of telmisartan and amlodipine combination on blood pressure, echocardiographic parameters and microalbuminuria in patients with metabolic syndrome". nephrology dialysis transplantation great clarendon st, oxford ox2 6dp, england: oxford univ press, 33 (2018): 374.

5. Alyavi B., et al. "Role of pro-inflammatory cytokines in metabolic syndrome”. Allergy 111 River St, Hoboken 07030-5774, Nj Usa: Wiley, 73 (2018): 562-562.

6. Andreeva EO. "Features of the functional state of the vascular wall endothelium and the nature of heterogeneous vasodilation in patients with coronary artery disease II-III functional class of angina of exertion". Siberian Medical Journal 3 (2008): 17-21.

7. Babaev M., et al. "P2533 Influence of l-arginine aspartate on vascular markers in hypertensive patients with metabolic syndrome”. European Heart Journal 39.1 (2018): 565. P2533.
8. Badger A.L. "The regulatory role of the endothelium and some aspects of the effect of pharmacotherapy on its function". Modern technologies in medicine 3 (2011): 142-146.

9. Blum A and Miller H. "Role of cytokines in heart failure". American Heart Journal 135.2-1 (1998): 181-186.

10. Brunner H. "Endothelial function and dysfunction. Part II: Association with cardiovascular risc factors and diseases. A statement by the working. Group on Endothelins and Endothelial Factors on the European society of Hypertension". Journal of Hypertension 23.2 (2005): 223-246.

11. Koziolov NA. "Assessment of vascular remodeling in patients with coronary heart disease, depending on the severity of chronic heart failure". Heart Failure 2.58 (2010): 83-88.

12. Kremneva LV. "Interleukin-6 and cell adhesion molecules: association with risk factors and prognosis of coronary heart disease". Clinical Pharmacology and Therapy 13 (2004): 7884.

13. Lutfullayevich AA., et al. "GW28-e0699 Cardiovascular risk stratification and gender differences in hypertensive patients with metabolic syndrome". Journal of the American College of Cardiology 70.16 (2017): C138-C139.

14. Lyutfullayevich AA., et al. "Relationship Between Hemodynamic Parametres And Nppa, Nppb, Npr3 Genes Polymorphism in Patients with Ischemic Heart Disease". International Scientific Review7.38 (2017).

15. Pan JP., et al. "The value of plasma levels of tumor necrosis factor-alpha and interleukin-6 in predicting the severity and prognosis in patients with congestive heart failure". Journal of the Chinese Medical Association 67.5 (2004) 222-228.

16. Parkhomenko Yu.V. "Features of the function of the ventricles of the heart and endothelium in gerontological patients with stable exertional angina during the formation of chronic heart failure". Zabay Medical Report 2.8 (2007): 12.

17. Shevchenko A Yu. "Endothelium - the structural basis of the circulatory system: the history of the problem". Bulletin of the National Medical and Surgical Center Nikolay Pirogov 6.2 (2011): 9-15. 
18. Sukmanova IA. "Indicators of endothelial function, morphofunctional parameters of the heart and metabolic status in diastolic heart failure in patients of different age groups". Cardiology and Cardiovascular Surgery 3 (2012): 72-75.

19. Usarov M. et al. "Ps 11-56 Efficacy of Combination Therapy Using Nebivalol And Trimetazidine In Hypertensive Patients with Metabolic Syndrome and Stable Angina". Journal of Hypertension 34 (2016): e349.

20. Uzokov J., et al. "Influence of combination therapy of rosuvastatin and telmisartan on vascular and metabolic profile in hypercholesterolemic patients with metabolic syndrome". Atherosclerosis 263 (2017): e241.

21. Wang J., et al. "Systolic and diastolic dyssynchrony in patients with diastolic heart failure and the effect of medical therapy". Journal of the American College of Cardiology 49.1 (2007): 8896.

\section{Volume 3 Issue 8 August 2019}

(C) All rights are reserved by DI Radjabova., et al. 